\title{
Robust and Low-Complexity Cooperative Spectrum Sensing via Low-Rank Matrix Recovery in Cognitive Vehicular Networks
}

\author{
Xia Liu (D), Zhimin Zeng, and Caili Guo \\ Beijing Laboratory of Advanced Information Networks, School of Information and Communication Engineering, \\ Beijing University of Posts and Telecommunications, Beijing, China \\ Correspondence should be addressed to Xia Liu; liuxiabupt@bupt.edu.cn
}

Received 7 February 2018; Revised 16 April 2018; Accepted 15 May 2018; Published 26 June 2018

Academic Editor: Mahdi Ben Ghorbel

Copyright (C) 2018 Xia Liu et al. This is an open access article distributed under the Creative Commons Attribution License, which permits unrestricted use, distribution, and reproduction in any medium, provided the original work is properly cited.

In cognitive vehicular networks (CVNs), many envisioned applications related to safety require highly reliable connectivity. This paper investigates the issue of robust and efficient cooperative spectrum sensing in CVNs. We propose robust cooperative spectrum sensing via low-rank matrix recovery (LRMR-RCSS) in cognitive vehicular networks to address the uncertainty of the quality of potentially corrupted sensing data by utilizing the real spectrum occupancy matrix and corrupted data matrix, which have a simultaneously low-rank and joint-sparse structure. Considering that the sensing data from crowd cognitive vehicles would be vast, we extend our robust cooperative spectrum sensing algorithm to dense cognitive vehicular networks via weighted low-rank matrix recovery (WLRMR-RCSS) to reduce the complexity of cooperative spectrum sensing. In the WLRMR-RCSS algorithm, we propose a correlation-aware selection and weight assignment scheme to take advantage of secondary user (SU) diversity and reduce the cooperation overhead. Extensive simulation results demonstrate that the proposed LRMR-RCSS and WLRMR-RCSS algorithms have good performance in resisting malicious SU behavior. Moreover, the simulations demonstrate that the proposed WLRMR-RCSS algorithm could be successfully applied to a dense traffic environment.

\section{Introduction}

Social problems of road accidents, traffic congestion, and air pollution are becoming increasingly severe with the increasing number of vehicles worldwide. According to a report published by the World Health Organization (WHO), approximately 1.25 million people die each year because of road traffic collisions [1]. Vehicular networks are envisioned to revolutionize the lifestyle of human beings within the next few years, with the aim of reducing the number of traffic collisions and providing entertainment services. According to the current standard for Wireless Access in Vehicular Environments (WAVE) [2, 3], multiple channels with one control channel $(\mathrm{CCH})$ and six service channels $(\mathrm{SCHs})$ for data exchange are permitted to support safety-related services (e.g., lane change assistance, and intersection collision warning) and non-safety-related services (e.g., commercial infotainment, multimedia downloads).

However, both theoretical analysis and simulation results indicate that the currently allocated bandwidth is not sufficient to provide reliable safety-related services under certain heavy traffic conditions [4-7]. The generation rate of a typical basic safety message (BSM) is from 2 to 10 messages per second to support many safety-related applications [6]. The high probability of an increased BSM generation rate in a heavy traffic environment will lead to the $\mathrm{CCH}$ becoming congested due to an increased number of packet collisions. This congestion will decrease the reliability of vehicular communication. Moreover, certain studies have demonstrated that non-safety-related services of the allocated band might also have to be severely restricted in highdensity traffic. Reference [7] proved that a large share of nonsafety-related services only appropriates in low or moderate traffic conditions. Additionally, only $10 \%$ of the bandwidth would remain for non-safety-related applications in order to guarantee $95 \%$ of the reliability of transmissions for safetyrelated applications in a high traffic environment.

Cognitive radio (CR) technology is a feasible measure that has been used to solve the spectrum scarcity problems in vehicular networks (see, e.g., the recent overviews in $[8,9]$ ). 
In cognitive vehicular networks (CVNs), as unlicensed users, the vehicles equipped with $\mathrm{CR}$ can detect and use other idle licensed spectrums when the primary user (PU) is absent. Cooperative spectrum sensing (CSS) has been extensively investigated in efforts to improve the detection performance via the diversity gain of cooperative secondary users (SUs) in CVNs [10-14]. These papers have shown that CSS can achieve spatial diversity gains under the assumption that the collaborative SUs are proactive. However, none of these studies have considered that SU sensing data may be unreliable due to either certain malicious behaviors or unexpected equipment failures. Many envisioned applications in vehicular networks that are related to safety would need high reliable connectivity. Therefore, some preliminary work in [15-20] has focused on increasing the robustness of cooperative spectrum sensing in cognitive vehicular networks. However, it is hard to implement these methods in practical CVNs environment due to their complexity, especially under heavy traffic conditions. While moving on the road, it is difficult to detect a malicious vehicle that may be transmitting untrustworthy spectrum sensing data during a sensing period. One challenge in CSS is the uncertainty of the sensing data quality, which may be corrupted by unreliable vehicles. This uncertainty motivated us to investigate the issue of efficient and robust CSS in CVNs. We formulate an optimization problem as a low-rank and sparse recovery by utilizing the real spectrum occupancy matrix and corrupted data matrix, which have a simultaneously low-rank and joint-sparse structure. In our previous work [21], our model simply assumed that cognitive vehicles carried out low-speed and single movement on a highway. But this assumption, apparently, is not always conformed with the real case, considering that, in CVNs, vehicle density reveals sparse and dense fluctuations with the space and time. As there are few users participating in cooperative sensing with sparse traffic flows, it is impossible to improve the detection probability of cooperative spectrum sensing. When the vehicles are dense, it has a large number of cooperative users, which makes the algorithm more expensive. In view of the above problems, this study is focused on establishing different algorithms for actual nondense and dense traffic environment. Firstly, LRMR-RCSS algorithm in this paper is established to recover the real data from noisy and corrupted data for improving the spectrum sensing data quality and CSS performance, which is applicable to low traffic density environments. The low-rank matrix $\mathbf{X}$ is directly recovered by the ALM algorithm. At the same time, we extend our robust cooperative spectrum sensing algorithm WLRMRRCSS algorithm into dense cognitive vehicular networks by considering the reliability of cooperative cognitive vehicles. Different from [21], this study concentrates on the adaptability of the WLRMR-RCSS algorithm with the change of traffic density. In this paper, we analyze the improvement of algorithm performance in traffic density from the sparse to dense state and the changes in the number of selected cooperative users under different traffic density. In the simulation process, VISSIM, the software of traffic flow, is used to generate the traffic flow in this article. We demonstrate that our proposed LRMR-RCSS and WLRMR-RCSS algorithms are secure and more efficient in CVNs, and the
WLRMR-RCSS algorithm is particularly robust against traffic density changes.

1.1. Related Work. In traditional CSS, many defense methods have been proposed in the literature in order to mitigate the negative effect of false spectrum sensing data. These methods could be classified into SU weighting schemes and SU filtering schemes. In SU weighting schemes, all the spectrum sensing data take part in the cooperation and smaller weights are assigned to the data of lower quality. In SU filtering schemes, it is to take out the "detected malicious SUs," and only utilize the remaining spectrum sensing data from the "detected honest SUs" for cooperation.

In the context of SU weighting schemes in CVNs, paper [15] proposed to apply Belief Propagation (BP) in order to establish the belief on the existence of primary users to its neighbors. In this paper, the spatial correlation between neighboring vehicles is exploited by message passing. In [16], an entropy-based voting algorithm was proposed to decide whether a channel is available with its one-hop neighbor vehicles. In [10], a weighting function based on the distance between the vehicle and its neighbor is established for the sake of evaluating neighbors' credibility with reference to the aggregated spectrum sensing decisions. In fact, the distance may not actually be in accordance with the neighbors' credibility. Due to fading of the communication links, environmental obstacles, or transmission errors, each neighbor's credibility can potentially be distinct based on the vehicular environment. In addition, many security threats have been raised as a result of the openness of low-layer protocol stacks in cognitive vehicular networks [17]. The malicious cognitive vehicles can introduce false data to confuse the cooperated vehicles. At this point, CSS would be distorted by malicious cognitive vehicles. For example, when the false data is introduced, the CSS result might conclude in the presence of PU, on the contrary. By doing so, these malicious cognitive vehicles can use the PU channels selfishly. References $[18,19]$ introduced CSS with trust assistance to solve the security issue that was introduced by a spectrum sensing data falsification (SSDF) attack in CVNs. Despite few SU filtering schemes in CVNs, data recovery algorithm provides a new approach. In [20], in order to mitigate the influence of abnormal data on the performance of CSS, a robust cooperative spectrum sensing has been studied for a wireless sensor networks environment. Nuclear norm minimization is adopted to recover the real spectrum sensing data in this paper.

The aforementioned methods [15-19] have played a vital role in fostering new strategies for robust spectrum sensing in CVNs. However, many of the methods in these papers are trust-based, which utilize historical information on malicious vehicles' behavior. Responsible reputation information is not invariably available because well-established historical statistics would be too expensive or even unrealistic in fast changing CVNs. In addition, as [22] notes, intelligent malicious users can send random false values that are close to the real values. In this case, it is more of a challenge to recognize the malicious users than the types that always send very high or very low values. The aforementioned low-rank matrix 
recovery-based CSS method [20] focuses on improving sensing data quality without considering high data transmission cost in CSS networks. These above methods are complex to implement in practical CVNs due to their complexity or hardware facility. In CVNs, the network topology changes quickly with diverse vehicles taking part in the cooperation at different times. The cost of transmission from cognitive vehicles to the fusion center (FC) is high as all the collected cognitive vehicles' spectrum sensing data are transmitted to an FC, which is inefficient or even unaffordable. Therefore, a robust cooperative spectrum detection algorithm with low complexity in CVNs is strongly needed but challenging to achieve.

1.2. Our Contribution. In this paper, our contribution comprises three parts.

(i) We develop a robust cooperative spectrum sensing algorithm LRMR-RCSS in CVNs, with a view to guaranteeing sensing data quality. We recover the real spectrum sensing data from the noisy and abnormal data to improve the quality of the sensing data by taking advantage of the real spectrum occupancy matrix, and the corrupted data matrix has a simultaneously low-rank and joint-sparse structure.

(ii) By extending our LRMR-RCSS algorithm to dense CVNs, we propose a WLRMR-RCSS algorithm. In the WLRMR-RCSS algorithm, we establish a correlationaware selection and weight assignment scheme for cooperative SUs in heavy traffic environments. A certain number of cognitive vehicles are chosen by considering the correlation between the SUs. Based on this scheme, our WLRMR-RCSS algorithm obtains cooperative SU diversity and reduces cooperative overhead. The complexity of the cooperative spectrum sensing in the CVNs is reduced.

(iii) The simulations demonstrate that the LRMR-RCSS and WLRMR-RCSS algorithms can effectively mitigate the adverse effects of corrupted data introduced by the malicious behaviors of SUs. Moreover, the proposed WLRMR-RCSS algorithm can be successfully applied in a dense traffic environment.

The remainder of this paper is organized as follows. In Section 2, we construct the system model and problem formulation. In Section 3, we provide details of our proposed LRMR-RCSS and WLRMR-RCSS algorithms. In Section 4, we present the simulation results and demonstrate the correctness of the theoretical analysis and make comparisons with other algorithms under low and high traffic density environments. Finally, we provide our conclusions in Section 5. Table 1 shows the notations.

\section{System Model and Problem Formulation}

2.1. System Model. A typical cognitive vehicular network communications scenario is shown in Figure 1. For simplicity, we consider a cognitive vehicular network scenario of a road that consists of a primary network and a secondary
TABLE 1: Notations.

\begin{tabular}{|c|c|}
\hline Variables & Explanation \\
\hline $\mathrm{PU}$ & Primary user \\
\hline SU & Secondary user \\
\hline CVNs & Cognitive vehicular networks \\
\hline CSS & Cooperative spectrum sensing \\
\hline FC & Fusion center \\
\hline ED & Energy detection \\
\hline SSDF & spectrum sensing data falsification \\
\hline EGC & Equal Gain Combining \\
\hline ADMM & The proximal alternating direction method of multiplier \\
\hline$P_{d}$ & The detection probability \\
\hline$P_{f}$ & The false alarm probability \\
\hline$r_{m}$ & the received signal at the $m$ th SU in one subband \\
\hline$T h$ & The preset threshold at the FC \\
\hline$y_{m}$ & The detected energy at the $m$ th SU \\
\hline$Y_{n}$ & The detected energy calculated by EGC \\
\hline$\sigma_{s, m}^{2}$ & The received primary signal power at the $m$ th SU \\
\hline$\sigma_{n}^{2}$ & The noise power \\
\hline$h_{m}$ & The channel response \\
\hline$d_{\text {corr }}$ & The decorrelation function \\
\hline$R(\cdot, \cdot)$ & The correlation function between two nodes \\
\hline$d_{i j}$ & The distance between the node $i$ and $j$ \\
\hline$\Omega$ & The set of vehicles \\
\hline$N$ & The number of subband \\
\hline$N_{\text {sam }}$ & The number of samples in each subband \\
\hline$\lambda$ & The tradeoff parameter \\
\hline$\mu$ & A constant $(>0)$ \\
\hline$M$ & The number of cooperative vehicles \\
\hline$\rho$ & A constant $(>1)$ \\
\hline$s_{t}(\cdot)$ & The shrinkage operator \\
\hline $\mathrm{Y}$ & The sensing data matrix at the FC \\
\hline $\mathbf{R}$ & The energy matrix \\
\hline $\mathbf{O}$ & The real occupancy state matrix \\
\hline A & The energy detector output matrix \\
\hline $\mathrm{V}$ & The noise matrix \\
\hline A & The corrupted data matrix \\
\hline W & The weighting matrix \\
\hline $\mathrm{U}$ & The unitary matrix \\
\hline$\Sigma$ & The positive semi-definite diagonal matrix \\
\hline $\mathbf{Q}$ & The unitary matrix \\
\hline G & The Lagrangian multiplier matrix \\
\hline
\end{tabular}

network that is colocated in a geographical area. The PU owns several licensed wideband channels that are divided into $N$ subbands. According to the existing spectrum measurements in [24-26], the licensed subbands are underutilized; namely, the spectrum occupancy rate of these $N$ subbands is relatively low. The secondary network is an infrastructurebased network that contains several SUs and a fusion center. 
There are many vehicles traveling along the road, and they are equipped with spectrum sensing-enabled terminals and Global Positioning System (GPS) devices. M SUs participate in the cooperative sensing process. Furthermore, the SUs are supposed to be randomly distributed along the road. Each SU first performs energy detection (ED) to detect the presence of a PU in each subband, either concurrently [27] or sequentially [28], and then reports the measurement results to the FC at the end of the sensing period. In the case of sequential sensing, each SU has to report the measurements to the FC which should collect the measurements from all SUs. This process may take time, especially under high traffic environment. By the time the FC took the decision, the SU might have a different observation. Therefore, we adopt the pattern of [27] in this paper. After fusing the collected sensing data, the FC makes a decision regarding the occupancy state of the $N$ subbands as either present $\left(H_{1}\right)$ or absent $\left(H_{0}\right)$. It is further noticed that some of the Sus would send corrupted values to the $F C$, such as $S U_{1}$, $S U_{4}$, and $S U_{M}$, as labeled in Figure 1. Because some SUs may experience deep fading or shadowing in CVNs, they may always send very low power values to the $\mathrm{FC}$ regardless of the spectrum occupancies. On the other hand, some SUs may show malicious behaviors in order to use the PU channels selfishly. These malicious vehicles appear randomly to corrupt a random number of channels at random locations.

Assume that the PU state remains unchanged during each spectrum sensing period. We adopt ED; then, the received signal $r_{m}$ at the $m$ th SU in one subband under the two hypotheses is expressed as

$$
\begin{aligned}
r_{m}(k)= \begin{cases}n_{m}(k), & H_{0} \\
h_{m} s_{m}(k)+n_{m}(k), & H_{1},\end{cases} \\
\quad k=0,1, \ldots, N_{\text {sam }}-1
\end{aligned}
$$

where $N_{\text {sam }}$ is the number of samples in each subband, $n_{m}$ is additive white Gaussian noise (AWGN) with zero mean and variance $\sigma_{n}^{2}, h_{m}$ is the channel response related to the location of the SU, and $s_{m}$ denotes the transmitted signal of the PU. The detected energy $y_{m}$ at the $m$ th SU is

$$
y_{m}=\frac{1}{N_{\text {sam }}} \sum_{k=0}^{N_{s a m}-1}\left|r_{m}(k)\right|^{2}
$$

According to the Central Limit Theorem, $y_{m}$ can approximate the Gaussian distribution as

$$
y_{m} \sim \begin{cases}N\left(\sigma_{n}^{2}, \frac{2 \sigma_{n}^{4}}{N_{s a m}}\right) & H_{0} \\ N\left(\left(\sigma_{n}^{2}+\sigma_{s, m}^{2}\right), \frac{2\left(\sigma_{n}^{2}+\sigma_{s, m}^{2}\right)^{2}}{N_{s a m}}\right) & H_{1}\end{cases}
$$

where $\sigma_{s, m}^{2}$ is the received primary signal power and $\sigma_{n}^{2}$ is the noise power.
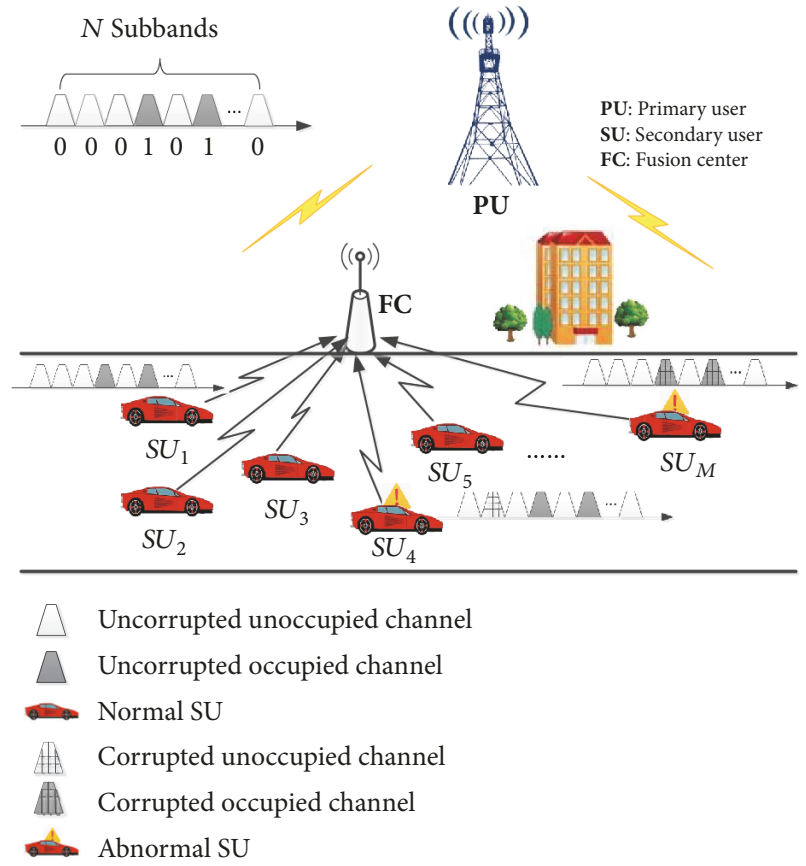

FIGURE 1: Network model of cooperative spectrum sensing in CVNs with malicious users.

After performing local spectrum sensing, SUs send their local ED results directly to the FC. For the soft-decision schemes [29] considered in this paper, the FC employs the equal gain combining (EGC) rule and calculates the ED results $Y_{w}$. According to (2) and (3), $Y_{w}$ obeys the distribution

$$
\begin{aligned}
& Y_{w} \\
& \sim \begin{cases}N\left(M \sigma_{n}^{2}, \frac{2 M \sigma_{n}^{4}}{N_{s a m}}\right) & H_{0} \\
N\left(\sum_{m=1}^{M}\left(\sigma_{n}^{2}+\sigma_{s, m}^{2}\right), \sum_{m=1}^{M} \frac{2\left(\sigma_{n}^{2}+\sigma_{s, m}^{2}\right)^{2}}{N_{s a m}}\right) & H_{1}\end{cases}
\end{aligned}
$$

Then, the detection probability $P_{d}$ and false alarm probability $P_{f}$ can be expressed as

$$
\begin{aligned}
P_{d} & =P\left(Y_{w}>T h \mid H_{1}\right) \\
& =Q\left(\frac{T h-\sum_{m=1}^{M}\left(\sigma_{n}^{2}+\sigma_{s, m}^{2}\right)}{\sqrt{\sum_{m=1}^{M}\left(2\left(\sigma_{n}^{2}+\sigma_{s, m}^{2}\right)^{2} / N_{s a m}\right)}}\right) \\
P_{f} & =P\left(Y_{w}>T h \mid H_{0}\right)=Q\left(\frac{T h-\sigma_{n}^{2}}{\sqrt{2 \sigma_{n}^{4} / N_{s a m}}}\right)
\end{aligned}
$$


where $Q(x)$ is the complementary distribution function, and $Q(x)=(1 / \sqrt{2 \pi}) \int_{x}^{+\infty} \exp \left(-x^{2} / 2\right) d x$. Th represents the preset threshold in the FC,

$$
T h=\sqrt{\frac{2 \sigma_{n}^{4}}{N_{s a m}}} Q^{-1}\left(P_{f}\right)+\sigma_{n}^{2}
$$

where $Q^{-1}(\cdot)$ is the inverse $Q$-function.

Note that the distance between SUs and a PU is a crucial parameter for spectrum sensing in CVNs because the distance determines whether a $\mathrm{PU}$ is inside the sensing range of SUs or not. Because cognitive vehicle is mobile, the PU may fall within or outside the sensing range of the cognitive vehicle after a certain time. Whether or how long the PU in CVNs can fall within the SUs' sensing range is determined by the speed and direction of the cognitive vehicle. Different from other mobile ad hoc networks, cognitive vehicles in CVNs move in same direction or opposite directions based on the road structure. It has demonstrated that whether a PU is inside the sensing range of SUs would be related to velocity of SUs, sensing range of SU, and transmission range of $\mathrm{PU}$ $[30,31]$.

2.2. Problem Formulation. Although cooperation can significantly exploit the spectrum sensing in CVNs, it also introduces a security hole for various malicious attackers. Some studies have recently considered an attack model known as SSDF [32-34], which is a fatal threat to CSS. There are several typical attack patterns under the SSDF model, which are known as always opposite ( $\mathrm{AO})$, always busy ( $\mathrm{AB})$, always free $(\mathrm{AF})$, and random disguising. In these attack models, $\mathrm{AO}$ attacks always send opposite decisions. $\mathrm{AB}$ attacks always report that the $\mathrm{PU}$ is present, whereas $\mathrm{AF}$ attacks always declare that the PU is absent. The random disguising attack may not always transmit false spectrum sensing data.

Notably, all of these attack patterns introduce corrupted spectrum sensing data for CSS. Figure 2 shows an example of a corrupted data distribution in CVNs. Some vehicles may nonrandomly transmit fake spectrum sensing data as $S_{4}$ (which always exhibit malicious behavior). Some vehicles may sporadically send unreliable data, such as $S U_{1}$ and $S U_{M}$. As in the above discussion, both nonrandom and random abnormal data have an adverse influence on the certainty of the spectrum sensing result. These events occur only occasionally in practice. In other words, the abnormal data are randomly and sparsely distributed. The matrix constructed by the received signals exhibits a low-rank property, as indicated in [35-37].

In this paper, we consider the case that every cognitive vehicle could sporadically and randomly contribute with abnormal data, due to either accidental equipment failures or random malicious behaviors, which makes the cooperative spectrum sensing inaccurate. Let $\mathbf{O}_{N \times N}:=\left[O_{n, n}\right], \mathbf{Y}_{M \times N}:=$ $\left[y_{m, n}\right], \mathbf{R}_{M \times N}:=\left[r_{m, n}\right], \mathbf{V}_{M \times N}:=\left[v_{m, n}\right]$, and $\mathbf{A}_{M \times N}:=$ $\left[a_{m, n}\right]$ denote the real occupancy state matrix, the sensing data matrix at the FC, the energy matrix for $M$ Sus, the

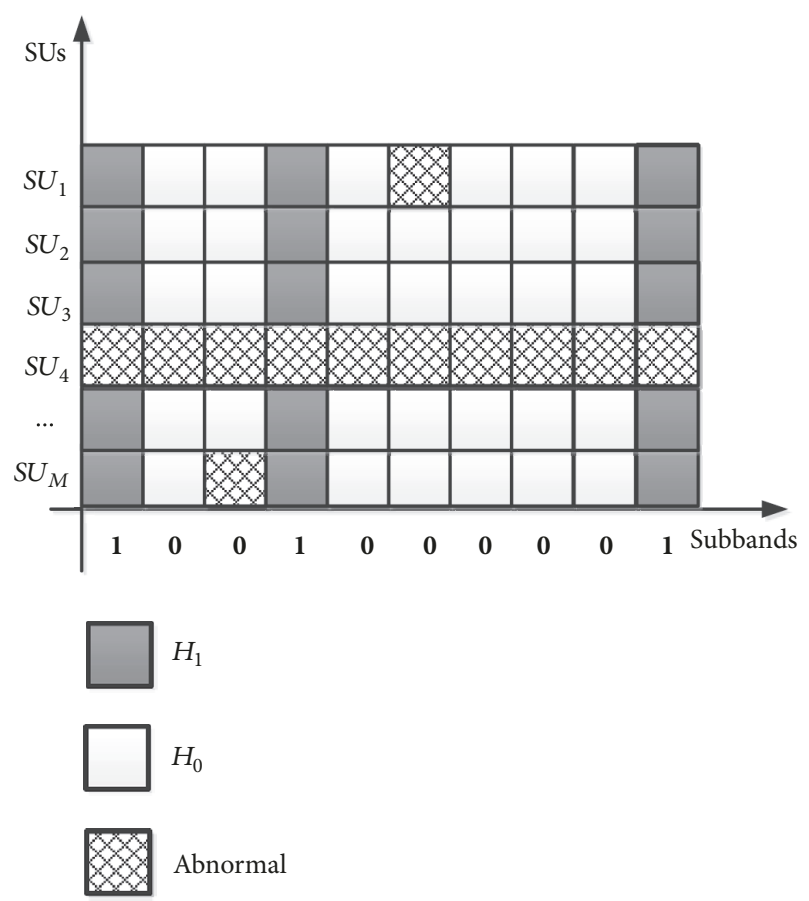

FIgURE 2: Example of a corrupted data distribution [21].

noise matrix, and the corrupted data matrix, respectively. The sensing data matrix $\mathbf{Y}$ at the FC can be expressed as

$$
\mathbf{Y}_{M \times N}=\mathbf{R}_{M \times N} \mathbf{O}_{N \times N}+\mathbf{V}_{M \times N}+\mathbf{A}_{M \times N}
$$

Diagonal matrix $\mathbf{O}$ satisfies $\operatorname{rank}(\mathbf{O})<N$ due to the underutilization of licensed bands. Therefore, matrix $\mathbf{O}$ is low-rank. Considering the random malicious behaviors in CVNs, nonzero entries in the matrix $\mathbf{A}$ are supposed to be randomly and sparsely distributed. So the matrix $\mathbf{A}$ has sparsity property. Our goal is to recover the real spectrum occupancy state matrix $\mathbf{O}$ from the noisy and corrupted observations $\mathbf{Y}$ by taking advantage of the low-rank property of $\mathbf{O}$ and the sparsity property of $\mathbf{A}$.

In static environment, malicious SUs would have dominated the location reliability for the fixed Region. However, in CVNs, the malicious cognitive vehicles distributed across all the road over time. On the other hand, mobile honest cognitive vehicles help to train location reliability of each road segment. As a result, the reports generated from a road segment at different times are from different vehicles. As malicious users are full of sparse characteristics, spectrum sensing data polluted in the report should also possess sparse features.

\section{Proposed Algorithms}

3.1. Robust Cooperative Spectrum Sensing via Low-Rank Matrix Recovery in CVNs (LRMR-RCSS). Here, we introduce a matrix $\mathbf{X}_{M \times N}:=\mathbf{R}_{M \times N} \mathbf{O}_{N \times N}$ that represents the energy detector output matrix. Matrix $\mathbf{X}$ is also low-rank because $\operatorname{rank}(\mathbf{X}) \leq \min (\operatorname{rank}(\mathbf{R}), \operatorname{rank}(\mathbf{O}))$. In such a CSS network, we must reconstruct the real energy matrix from the sensing 
data matrix at the FC by a low-rank matrix recovery technique [38-41]. The goal of recovering the spectrum occupancy state matrix $\mathbf{O}$ translates into approximately recovering matrix $\mathbf{X}$ because it is difficult to recover $\mathbf{O}$ directly.

According to the current low-rank matrix recovery theory, to recover the low-rank matrix $\mathbf{X}$ from the sensing data matrix $\mathbf{Y}$, it can be formulated as

$$
\begin{array}{ll}
\min _{\mathbf{X}, \mathbf{A}} & \operatorname{rank}(\mathbf{X})+\lambda\|\mathbf{A}\|_{0} \\
\text { s.t. } & \mathbf{Y}=\mathbf{X}+\mathbf{A}+\mathbf{V}
\end{array}
$$

where $\operatorname{rank}(\cdot)$ is the rank of the matrix, and $\|\cdot\|_{0}$ is the number of nonzero entries in the matrix. $\lambda$ is a positive rank-sparsity controlling parameter which represents a tradeoff parameter to balance matrix $\mathbf{X}$ and matrix $\mathbf{A}$.

According to previous research [39-41], we introduce a matrix $\mathbf{G}$ of the Lagrangian multiplier; then, model (9) could be transferred to minimizing the following augmented Lagrangian function $\mathscr{L}$ :

$$
\begin{aligned}
\mathscr{L}(\mathbf{X}, \mathbf{A}, \mathbf{G}, \mu)= & \|\mathbf{X}\|_{*}+\lambda\|\mathbf{A}\|_{1}+\langle\mathbf{G}, \mathbf{Y}-\mathbf{X}-\mathbf{A}\rangle \\
& +\frac{\mu}{2}\|\mathbf{Y}-\mathbf{X}-\mathbf{A}\|_{F}^{2}
\end{aligned}
$$

where $\|\cdot\|_{*}$ is the sum of the singular values and represents the nuclear norm of a matrix, $\|\cdot\|_{1}$ is the $l_{1}$-norm which denotes the sum of the absolute values of matrix entries, $\|\cdot\|_{F}$ is the Frobenius norm $\|\mathbf{A}\|_{F}=\sqrt{\operatorname{tr} \mathrm{AA}^{T}},\langle\mathbf{A}, \mathbf{B}\rangle \equiv \operatorname{tr}\left(\mathbf{A}^{\mathrm{T}} \mathbf{B}\right)$. Then, the optimal solution to the original problem can be obtained by iterating the following two steps until convergence for some $\rho>1$ :

$$
\begin{aligned}
\left(\mathbf{X}_{k+1}, \mathbf{A}_{k+1}\right) & \longleftarrow \min _{\mathbf{X}, \mathbf{A}} \mathscr{L}\left(\mathbf{X}, \mathbf{A}, \mathbf{G}_{k}, \mu_{k}\right) \\
\mu_{k+1} & \longleftarrow \rho \mu_{k} \\
\mathbf{G}_{k+1} & \longleftarrow \mathbf{G}_{k}+\mu_{k}\left(\mathbf{Y}-\mathbf{X}_{k+1}-\mathbf{A}_{k+1}\right)
\end{aligned}
$$

where $\mu>0$ denotes the penalty for infeasible points. According to [38], update the $\mathbf{X}$ and $\mathbf{A}$ in order to search for the optimal $\mathbf{X}$ and $\mathbf{A}$ alternately and iteratively as follows:

$$
\begin{aligned}
\mathbf{X}_{k+1}= & \arg \min \left(\mathbf{X}_{k}, \mathbf{A}_{k}, \mathbf{G}_{k}, \mu\right) \\
= & \arg \min \|\mathbf{X}\|_{*}+\frac{\mu_{k}}{2}\|\mathbf{Y}-\mathbf{X}-\mathbf{A}\|_{F}^{2} \\
& -\operatorname{Tr}\left(\left(\mathbf{G}_{k}\right)^{T} \mathbf{X}\right) \\
\mathbf{A}_{k+1}= & \arg \min \lambda\|\mathbf{A}\|_{*}+\frac{\mu_{k}}{2}\left\|\mathbf{Y}-\mathbf{X}_{k+1}-\mathbf{A}\right\|_{F}^{2} \\
& -\operatorname{Tr}\left(\left(\mathbf{G}_{k}\right)^{T} \mathbf{A}\right)
\end{aligned}
$$

Then the optimization problems in (11) can be solved as follows [41]:

$$
\begin{aligned}
& \mathbf{X}_{k+1} \longleftarrow \mathbf{U}_{k} \mathbf{s}_{\mu_{k}^{-1}}\left[\mathbf{\Sigma}_{k}\right] \mathbf{Q}_{k}^{\mathrm{T}} \\
& \mathbf{A}_{k+1} \longleftarrow s_{\lambda \mu_{k}^{-1}}\left[\mathbf{Y}-\mathbf{X}_{k+1}+\mu_{k}^{-1} \mathbf{G}_{k}\right] \\
& \mathbf{G}_{k+1} \longleftarrow \mathbf{G}_{k}+\mu_{k}\left[\mathbf{Y}-\mathbf{X}_{k+1}-\mathbf{A}_{k+1}\right]
\end{aligned}
$$

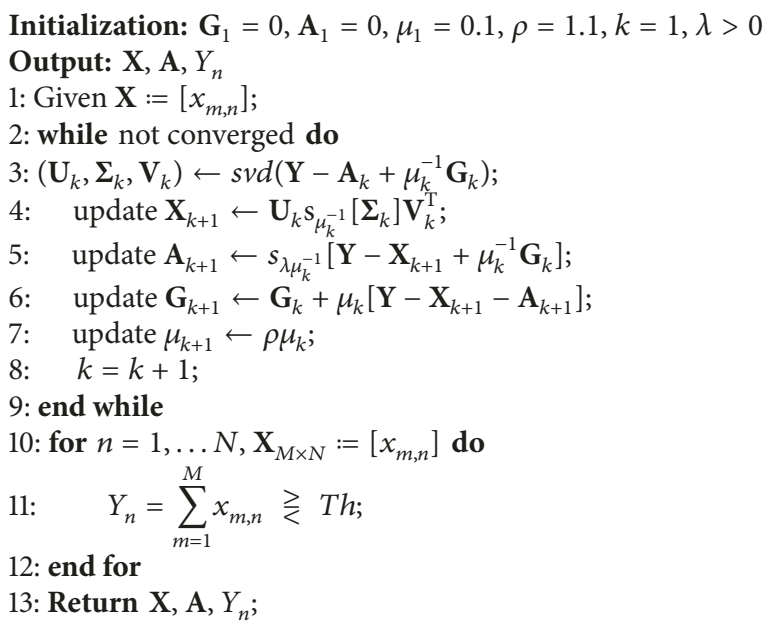

Algorithm 1: Robust cooperative spectrum sensing via low rank matrix recovery in CVNs (LRMR-RCSS).

where $s_{t}(\cdot)$ is shrinkage operator, which is defined as $s_{t}(x)=$ $\operatorname{sign}(x) \cdot \max (|x|-t, 0)$, and $\left(\mathbf{U}_{k}, \boldsymbol{\Sigma}_{k}, \mathbf{Q}_{k}\right) \equiv \operatorname{svd}\left(\mathbf{Y}-\mathbf{A}_{k}+\right.$ $\left.\mu_{k}^{-1} \mathbf{G}_{k}\right)$. We obtain the real sensing data $\mathbf{X}$ based on these steps. Next, we perform spectrum sensing by data fusion. The main steps of our proposed LRMR-RCSS are outlined in Algorithm 1.

Computational Complexity Analysis. The primary computational cost of the LRMR-RCSS algorithm is the singular value decomposition (SVD) of an $M \times N$ matrix in the process of updating $\mathbf{X}$ when using the augmented Lagrangian multiplier (ALM) approach. Its computational complexity is $O(M N \min (M, N))$. On the other hand, the number of iterations taken by the LRMR-RCSS algorithm to optimality is less vulnerable to changes in dimension, which has a worstcase complexity result of $O\left(k^{-2}\right)$.

\subsection{Extension to Robust Cooperative Spectrum Sensing} via Weighted Low-Rank Matrix Recovery in Dense CVNs (WLRMR-RCSS). In general, the calculation to solve the lowrank model is very large when faced with a large matrix problem. Reference [40] proposed an algorithm to reduce the complexity, but this algorithm must dynamically predict the value of the rank. Therefore, it suffers from high computational cost when $M$ is large during dense traffic in vehicular networks. In this part, we focus on solving the problem of robust CSS in a dense vehicular network environment. Cognitive vehicles experience different environmental conditions and contribute to spatial diversity based on their distribution. The sensing data could be combined based on the reliability factor of an SU, which is considered its weight. Therefore, we propose a novel weighted low-rank matrix recovery model for spectrum sensing as follows:

$$
\begin{array}{ll}
\min _{\mathbf{X}, \mathbf{A}} & \operatorname{rank}(\mathbf{X})+\lambda\|\mathbf{A}\|_{0} \\
\text { s.t. } & \mathbf{W} \circ \mathbf{Y}=\mathbf{W} \circ \mathbf{X}+\mathbf{V}+\mathbf{A}
\end{array}
$$




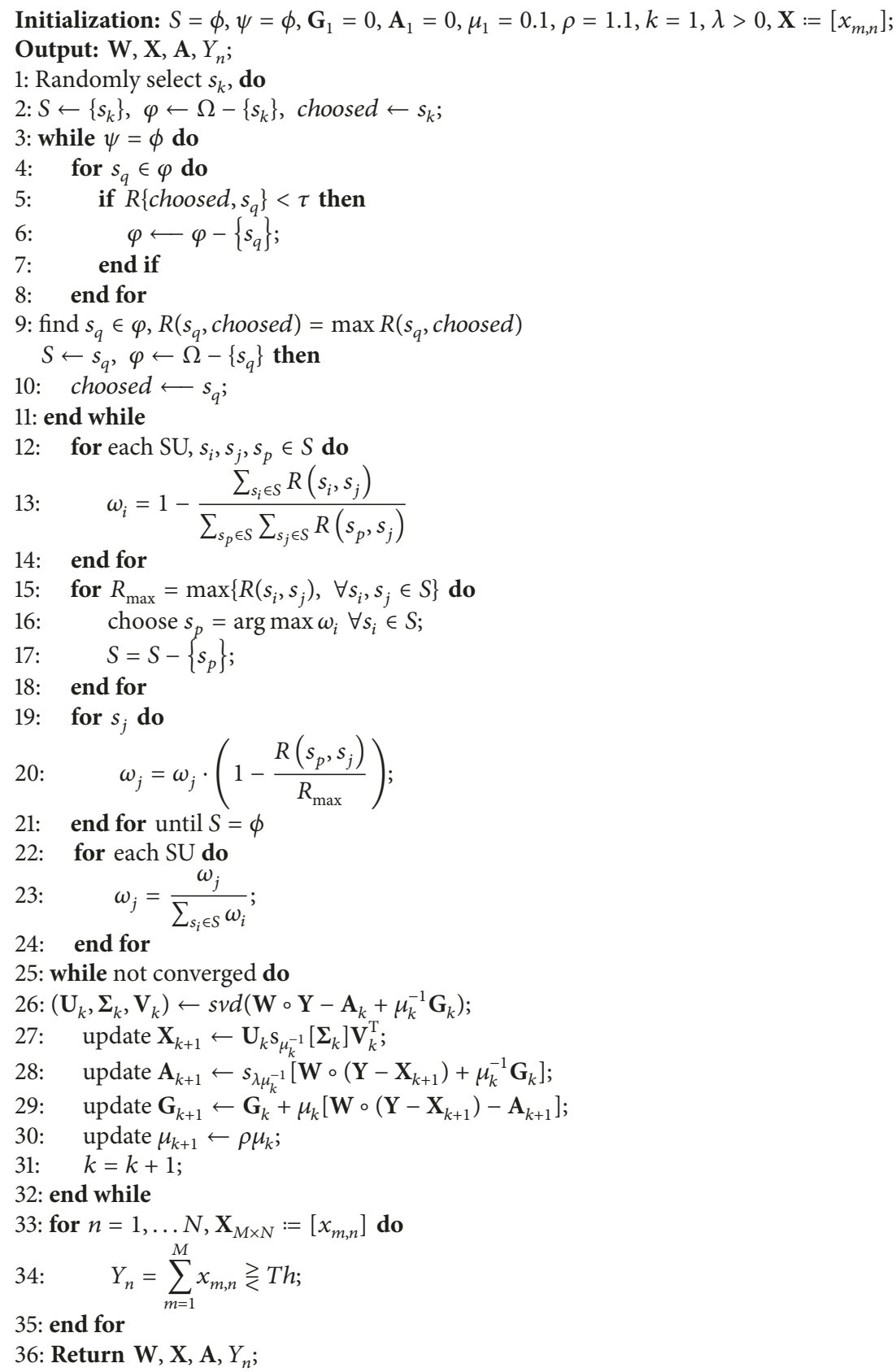

Algorithm 2: Robust cooperative spectrum sensing via weighted low rank matrix recovery in CVNs (WLRMR-RCSS).

where $\circ$ denotes the element-wise multiplication of two matrices and $\mathbf{W}$ is the weighting matrix which we will introduce in the following section. To address the aforementioned issues, the problem could typically be relaxed by tractable convex optimization, and it can be efficiently solved by the proximal alternating direction method of multipliers (ADMM) [38]. $\operatorname{rank}(\cdot)$ and $l_{0}$-norm are typically convex relaxed by the nuclear norm and $l_{1}$-norm.

$$
\begin{array}{ll}
\min _{\mathbf{X}, \mathbf{A}} & \|\mathbf{X}\|_{*}+\lambda\|\mathbf{A}\|_{1} \\
\text { s.t. } & \mathbf{W} \circ \mathbf{Y}=\mathbf{W} \circ \mathbf{X}+\mathbf{V}+\mathbf{A}
\end{array}
$$

Our model extends the classic matrix recovery model by considering the reliability of cooperative cognitive vehicles. Next we describe our proposed algorithm WLRMR-RCSS whose main steps are outlined in Algorithm 2.

\subsubsection{The Weighted Matrix $\mathbf{W}$ Establishment}

Step I (correlation-aware SU selection). First, we define the set $\Omega$ as all the vehicles in the road segment. We randomly selected $s_{k}$ among the set of vehicles $\Omega$. The set $\varphi$ is defined as the set of the remaining vehicles $\Omega-\left\{s_{k}\right\}$. According to [36], 


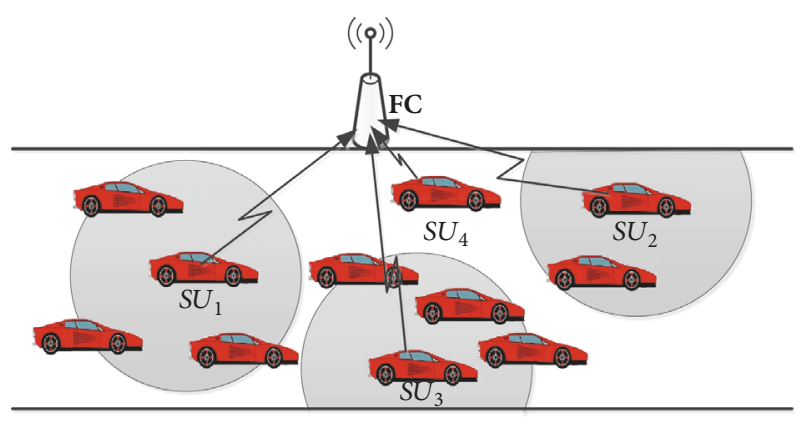

Figure 3: Example Scenario for Choosing Cooperative SUs (figure adapted from [21].).

we calculate the correlation function between $s_{k}$ and the other vehicles in set $\varphi$ :

$$
R\left(s_{k}, s_{q}\right)=e^{-\left(d_{k q} / d_{c o r r}\right) \cdot \ln 2}
$$

where $d_{k q}$ is the distance between the samples $s_{k}$ and $s_{q}$ and $d_{\text {corr }}$ is the decorrelation function. We set the decorrelation function to $20 \mathrm{~m}$ for urban environments as in [37]. We choose vehicle $s_{q}$, which satisfies $R\left(s_{k}, s_{q}\right)$, as the maximum among the vehicles in set $\varphi$. The vehicles with a correlation function smaller than a certain threshold $\tau$ are removed from set $\varphi$. Then, $s_{q}$ performs the steps presented above. This scheme is repeated until $\varphi \in \phi$. This correlation-aware SU selection scheme discards the correlated SU and selects the uncorrelated SUs among the remaining users. Finally, we denote the set of uncorrelated SUs with $S$. An example is shown in Figure 3. The subset $S$ which contains $S U_{1}, S U_{2}, S U_{3}$, and $\mathrm{SU}_{4}$ is established.

Step II (weight assignment). First, we calculate the weight $\omega_{i}$ of $s_{i}$ in set $S$.

$$
\omega_{i}=1-\frac{\sum_{s_{i} \in S} R\left(s_{i}, s_{j}\right)}{\sum_{s_{p} \in S} \sum_{s_{j} \in S} R\left(s_{p}, s_{j}\right)}
$$

We choose $s_{p}$ to satisfy $s_{p}=\arg \max \omega_{i}$. Then, we recalculate the weight of the remaining SUs using the equation $\omega_{j}^{\prime}=$ $\omega_{j} \cdot\left(1-R\left(s_{p}, s_{j}\right) / R_{\max }\right)$, where $R_{\max }=\max R\left(s_{i}, s_{j}\right)$. These steps are repeated for all $s_{j}$ in the set $S$. Finally, we normalize the weight $\omega_{j}=\omega_{j} / \sum_{s_{i} \in S} \omega_{i}$. Now, all of the cooperative SUs are assigned weights. To establish the weighting matrix $\mathbf{W}$ of cooperative SUs, we employ location to characterize the correlation function between the SUs and then establish a weight assignment scheme to transfer to a weighting matrix W. We divide this step into two phases to construct the weighting matrix; the weighted matrix $\mathbf{W}$ could be expressed as

$$
\mathbf{W}=\left[\begin{array}{ccc}
\omega_{1} & \cdots & \omega_{N} \\
\vdots & \cdots & \vdots \\
\omega_{1} & \cdots & \omega_{N}
\end{array}\right]_{M \times N}
$$

3.2.2. Robust Spectrum Sensing via Weighted Low-Rank Matrix Recovery. The authors of [20] proposed robust CSS with a crowd of low-end personal spectrum sensors. This paper assumed that all sensing data (both the normal data and the nonabnormal data) would be exploited for spectrum sensing to gain full diversity. As expressed in (15), our model extends the WLRMR-CSS to dense cognitive vehicular networks by taking the effect of correlation on aggregating the samples of SUs into consideration. The weighting matrix W assigns smaller weights to the corrupted data matrix in the sensing data matrix $\mathbf{Y}$; the $l_{1}$-norm of the corresponding vectors in the recovered sparse matrix $\mathbf{A}$ is inclined to be small. Thus, recovering the low-rank matrix $\mathbf{X}$ from $\mathbf{Y}$ can be highlighted more effectively.

Then, we focus on recovering the low-rank matrix $\mathbf{X}$ from Y. According to previous research [39-41], we introduce a matrix $\mathbf{G}$ of the Lagrangian multiplier; then, the solver of model (16) could instead be

$$
\begin{aligned}
\mathscr{L}(\mathbf{X}, \mathbf{A}, \mathbf{G}, \mu)= & \|\mathbf{X}\|_{*}+\lambda\|\mathbf{A}\|_{1} \\
& +\langle\mathbf{G}, \mathbf{W} \circ(\mathbf{Y}-\mathbf{X})-\mathbf{A}\rangle \\
& +\frac{\mu}{2}\|\mathbf{W} \circ(\mathbf{Y}-\mathbf{X})-\mathbf{A}\|_{F}^{2}
\end{aligned}
$$

Then, we can get the solutions as follows:

$$
\begin{aligned}
& \mathbf{X}_{k+1} \longleftarrow \mathbf{U}_{k} s_{\mu_{k}^{-1}}\left[\mathbf{\Sigma}_{k}\right] \mathbf{Q}_{k}^{\mathrm{T}} \\
& \mathbf{A}_{k+1} \longleftarrow s_{\lambda \mu_{k}^{-1}}\left[\mathbf{W} \circ\left(\mathbf{Y}-\mathbf{X}_{k+1}\right)+\mu_{k}^{-1} \mathbf{G}_{k}\right] \\
& \mathbf{G}_{k+1} \longleftarrow \mathbf{G}_{k}+\mu_{k}\left[\mathbf{W} \circ\left(\mathbf{Y}-\mathbf{X}_{k+1}\right)-\mathbf{A}_{k+1}\right]
\end{aligned}
$$

where $\left(\mathbf{U}_{k}, \boldsymbol{\Sigma}_{k}, \mathbf{Q}_{k}\right) \equiv s v d\left(\mathbf{W} \circ \mathbf{Y}-\mathbf{A}_{k}+\mu_{k}^{-1} \mathbf{G}_{k}\right)$. We obtain the real sensing data $\mathbf{X}$ based on these steps. Next, we perform spectrum sensing by data fusion.

Remark 1 . The set $\Omega$ is not fixed a priori but changes with the current vehicles within the road segment. The number of selected cognitive vehicles in the WLRMR-RCSS is able to adapt different traffic densities and different network topologies.

Remark 2. The proposed WLRMR-RCSS algorithm is centralized, and the FC handles the selection. This mechanism in the WLRMR-RCSS does not require explicit coordination communications among the SUs, thus limiting the communication overhead. During the selection process, the cognitive vehicles in $\Omega$ are all equipped with GPS devices. The FC could readily collect their location information.

Remark 3. The nonzero abnormal data might be generated by either accidental equipment failure or malicious behavior. The style of malicious behaviors could contain AO, AB, AF, or random disguising as previously discussed. The sparsity of these abnormal data is random during the process of spectrum sensing.

Remark 4. To recover all sensing data (both the normal data and the nonabnormal data) is time-consuming and 
complicated. In our Algorithm 2, both the normal sensing data and the nonabnormal sensing data of cooperated SUs will be separated. Meanwhile, the diversity of SUs is exploited for spectrum sensing.

Computational Complexity Analysis. The proposed WLRMRRCSS algorithm chooses $M$ noncorrelated vehicles with lower correlation coefficients and higher sensing reliability out of the total SUs. In this part, it needs $O\left(M^{2}\right)$ computational complexity. On the other hand, the reporting overhead will decline vastly without an obvious loss of performance with the SUs selection. For the part of robust spectrum sensing via weighted low-rank matrix recovery, its computational complexity is $O(M N \min (M, N))$. On the other hand, the WLRMR-RCSS algorithm has a worst-case complexity result of $O\left(k^{-2}\right)$, as is the case in LRMR-RCSS algorithm. The computational cost of the WLRMR-RCSS algorithm is not as high as [20] after the correlation-aware SUs selection step in our algorithm. Thus, our algorithm is efficient. BP-CSS in [15] must calculate the $k$ iterations of the exchange of information between vehicles, and its computational cost is $O\left(M^{k}\right)$. The overhead of the BlindCSS [42] is mainly from the transmissions made by all vehicles performing sensing of spectrum availability to all nearby RSUs. In ADMM-CSS [20], matrix $\mathbf{Y}$ is all of the spectrum sensing data of the SUs. As such, its computational complexity is more than that of our WLRMR-RCSS algorithm.

\section{Performance Evaluation}

Considering the actual vehicular environment, we analyze our algorithm in low and high traffic density environment scenarios. Our proposed algorithm simulation environment is shown in Figure 4. All of the mobility models in our simulations are generated using the software VISSIM [43]. We now evaluate our proposed schemes by comparing the ADMM-CSS algorithm [20], the BP-CSS algorithms in [15] (suitable for low traffic density), and the Blind-CSS algorithm [42] (suitable for high traffic density), as these schemes are all designed for robust cooperative spectrum sensing.

\subsection{Performance Analysis and Comparison}

Case I (low traffic density environment). To investigate our algorithm in a low traffic density environment, we consider a cognitive vehicular network on a highway, as shown in Figure 5. Detailed parameters are given in Table 2. We simulated $1000 \mathrm{~m}$ of a freeway. A primary transmitter is assumed in the middle, and crowd vehicles are randomly distributed. The Propagation Model computes the power received as in [10]. The rate of SUs that contribute abnormal data in all cooperated SUs is defined as $R_{\text {mal }}$. The $\rho_{\text {vel }}$ is the traffic density, which means the number of vehicles per meter per lane $(\mathrm{veh} / \mathrm{m} / \mathrm{l})$.

Figure 6 focuses on the influence of $R_{\text {mal }}$. A higher value of $R_{\text {mal }}$ indicates a higher scale of corrupted data. In this figure, although the sensing performance worsens with an

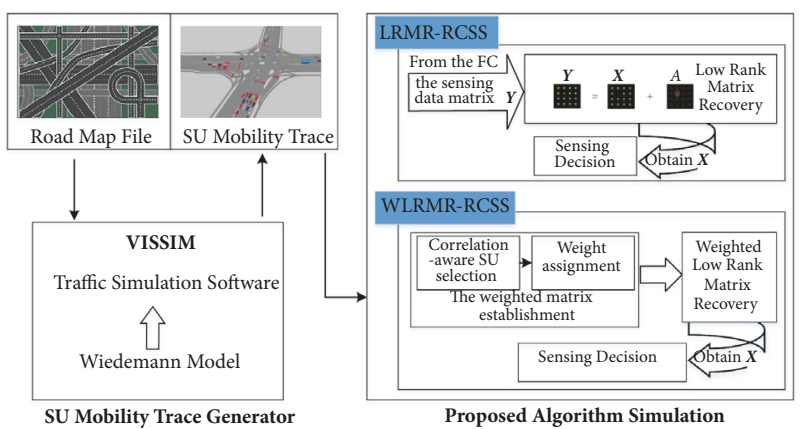

FIGURE 4: Our proposed algorithm simulation environment.

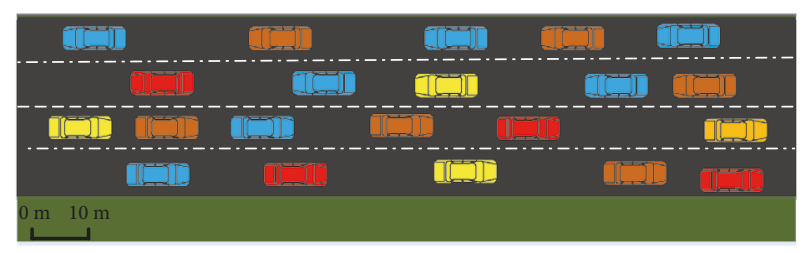

FIGURE 5: A cognitive vehicular network on a highway.

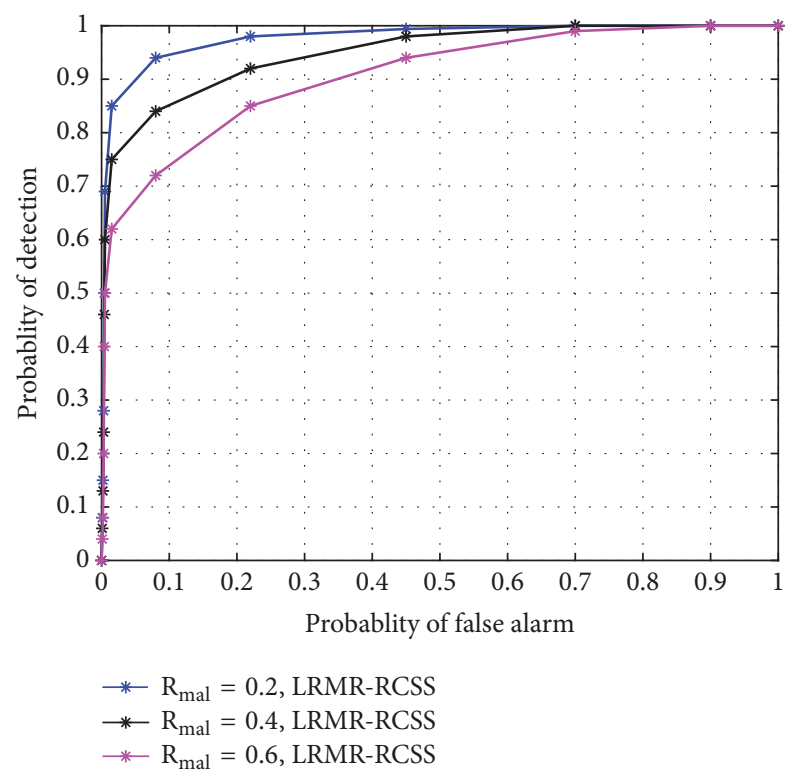

FIGURE 6: Sensing performance of various schemes under various abnormal data rates $R_{\text {mal }}$ for LRMR-RCSS [21].

increasing fraction of SUs that introduce corrupted data, our proposed LRMR-RCSS algorithm could achieve satisfactory performance in the case of a high number of malicious SUs.

As shown in Figure 7, to evaluate the performance of the proposed algorithm, we plot the complementary receiver operating characteristic (ROC) curve when $R_{\text {mal }}=0.1$. The simulation results in Figure 7(a) show that our proposed LRMR-RCSS algorithm has good performance in sparse traffic flow $\left(\rho_{v e l}=0.05\right)$. We find that LRMR-RCSS and WLRMR-RCSS algorithms both outperform the trust based among neighboring vehicles in BP-CSS, whether in a sparse 


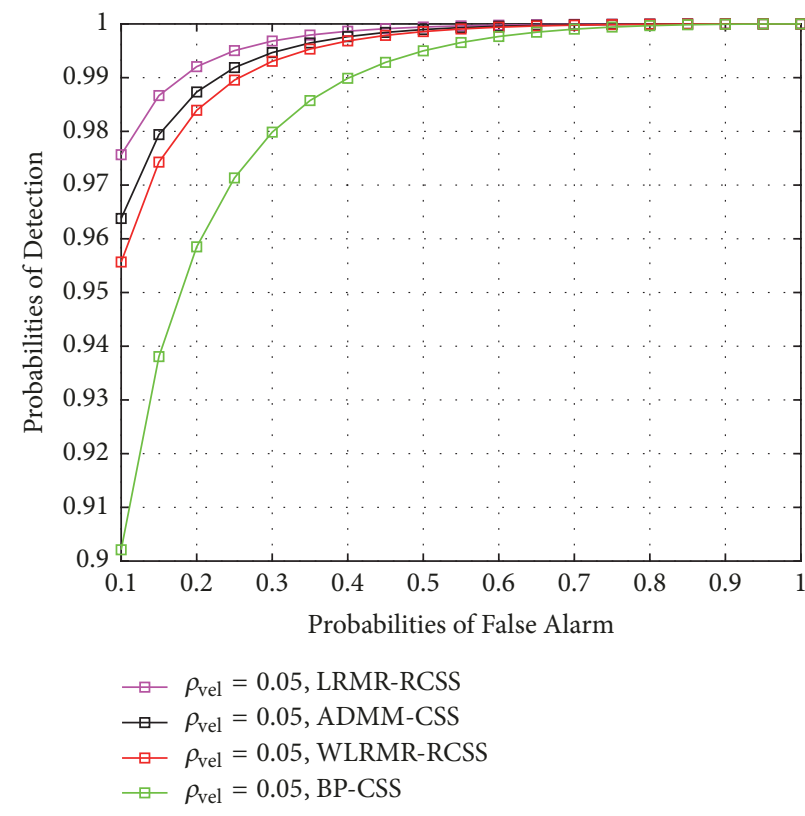

(a)

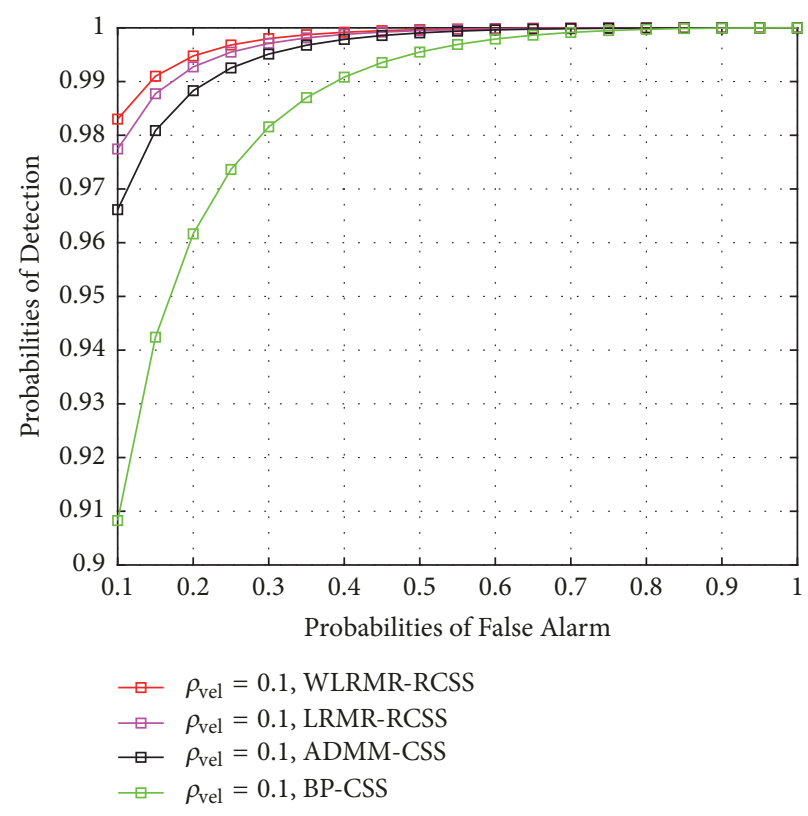

(b)

FIGURE 7: Comparison of the proposed LRMR-RCSS and WLRMR-RCSS algorithms with BP-CSS and ADMM-CSS algorithms at different traffic densities $\rho_{v e l}$.

TABLE 2: Simulation parameters.

\begin{tabular}{lc}
\hline Parameter & Value \\
\hline Road length (Highway) & $1000 \mathrm{~m}$ \\
\hline Road length & W-E: $1000 \mathrm{~m}$ \\
(Road intersection in Urban) & N-S: $800 \mathrm{~m}$ \\
\hline Lanes (Highway) & 4-lanes \\
\hline Lanes (Road intersection in Urban) & 4-lanes \\
\hline Traffic light timing & cycle $=100 \mathrm{~s}$ \\
(Road intersection in Urban) & red $=50 \mathrm{~s}$, green $=50 \mathrm{~s}$ \\
\hline Traffic flow (Highway) & $0-3000 \mathrm{veh} / \mathrm{h}$ \\
\hline Traffic flow & NS-SS: $0-1800 \mathrm{veh} / \mathrm{h}$ \\
(Road intersection in Urban) & ES-WS: $0-2700 \mathrm{veh} / \mathrm{h}$ \\
\hline Speed limit (Highway) & $50 \mathrm{~m} / \mathrm{s}(180 \mathrm{~km} / \mathrm{h})$ \\
\hline Speed limit (Urban) & $20 \mathrm{~m} / \mathrm{s}(72 \mathrm{~km} / \mathrm{h})$ \\
\hline SU mobility model & Wiedemann model $[23]$ \\
\hline Number of subbands $N$ & 20 \\
\hline Bandwidth of each subband BW & $200 \mathrm{kHz}$ \\
\hline Single/noise power & $27 \mathrm{dBm} /-110 \mathrm{dBm}$ \\
\hline$N_{\text {sam }}$ & 100 \\
\hline Sample time & $0.1 \mathrm{~ms}$ \\
\hline The path-loss exponent & $2-6$ \\
\hline shadowing dB-spread & $2-20 \mathrm{~dB}$ \\
\hline Simulation step for VISSIM & $0.1 \mathrm{~s}$ \\
\hline
\end{tabular}

or a moderate traffic flow environment. The traditional trust based cooperative spectrum sensing in cognitive vehicular networks is unable to determine the style of malicious SU behavior. For example, the malicious SUs may be considered

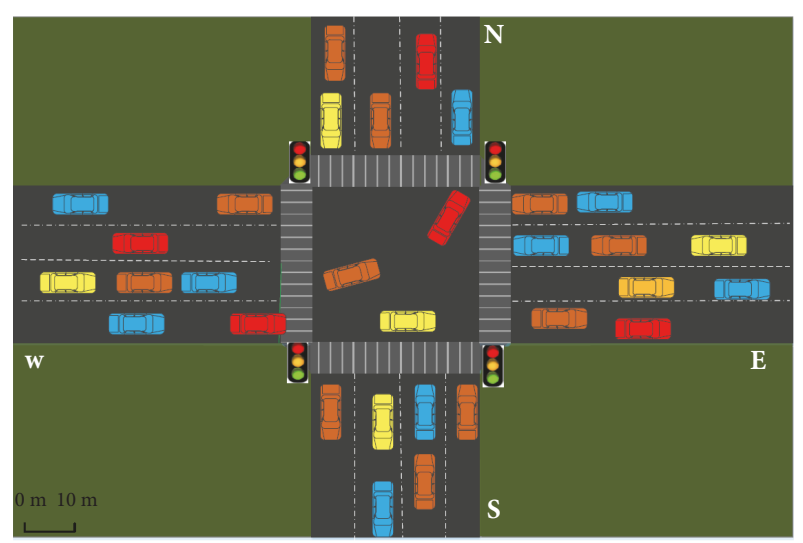

FIGURE 8: A cognitive vehicular network at a road intersection in an urban environment.

trustworthy when PU appears as if its form is AB attack. The inaccuracy due to inaccurate spectrum sensing data introduced by malicious SUs reduces the precision in BPCSS. From sparse to moderate, with an increase in the number of cooperative SUs, the performances of the three algorithms all increase due to the diversity effect of the cooperative SUs. The simulation results in Figure 7(b) show that our proposed WLRMR-RCSS algorithm could effectively eliminate the unreliable data component from the corrupted sensing data in moderate traffic densities.

Case II (high traffic density environment). To investigate our algorithm in a high traffic density environment, we consider a cognitive vehicular network at a road intersection with traffic lights in an urban area, as described in Figure 8. All vehicles 


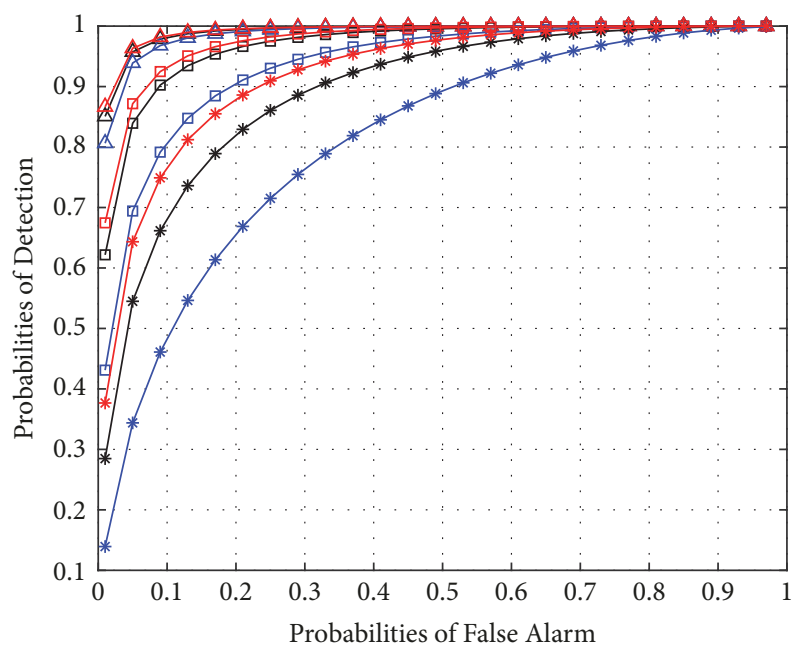

$$
\begin{aligned}
& \triangle \mathrm{R}_{\mathrm{mal}}=0.01, \text { ADMM-CSS } \\
& \triangle \mathrm{R}_{\mathrm{mal}}=0.01, \text { Blind-CSS } \\
& \square-\mathrm{R}_{\mathrm{mal}}=0.01, \text { WLRMR-RCSS } \\
& \square-\mathrm{R}_{\mathrm{mal}}=0.3, \text { ADMM-CSS } \\
& \square-\mathrm{R}_{\mathrm{mal}}=0.3, \text { Blind-CSS } \\
& \square-\mathrm{R}_{\mathrm{mal}}=0.3, \text { WLRMR-RCSS } \\
& *-\mathrm{R}_{\mathrm{mal}}=0.6, \text { ADMM-CSS } \\
& *-\mathrm{R}_{\mathrm{mal}}=0.6, \text { Blind-CSS } \\
& *-\mathrm{R}_{\mathrm{mal}}=0.6, \text { WLRMR-RCSS }
\end{aligned}
$$

FIGURE 9: Comparison of the proposed WLRMR-RCSS algorithm with Blind-CSS and ADMM-CSS algorithms with different $R_{\text {mal }}$ values.

could repeatedly move from one end to the other three ends. For example, among the vehicles departing from the left end, vehicles in the middle lane will turn left (toward the top end) at the intersection and vehicles in the right lane will go straight (toward the right end) at the intersection. The dense vehicle traffic at the road intersection in the urban area obeys the Wiedemann model generated by VISSIM.

As shown in Figure 9, our proposed WLRMR-RCSS algorithm has high performance in accordance with the ADMM-CSS algorithm when the value $R_{\text {mal }}$ is small. The Blind-CSS algorithm for vehicular networks is to be used for sharing with the surrounding vehicles. The choice of vehicles to perform the sensing operation is determined by vehicle speed. The Blind-CSS algorithm also has good performance because the SUs received few abnormal data. With the increase of the rate of abnormal data in all cooperating SUs sensing data, our algorithm is better than the ADMM-CSS algorithm because the weighting matrix in our algorithm assigns smaller weights to the corrupted data matrix in the sensing data matrix; then the corresponding vectors in the recovered real data matrix are inclined to be small. Our recovery of the real data matrix from the corrupted data matrix becomes more effective. The experimental results show that our WLRMR-RCSS algorithm is effective along with an increase in $R_{\text {mal }}$. The performance of the traditional blind cooperative sensing scheme as in Blind-CSS in CVNs would be influenced significantly.
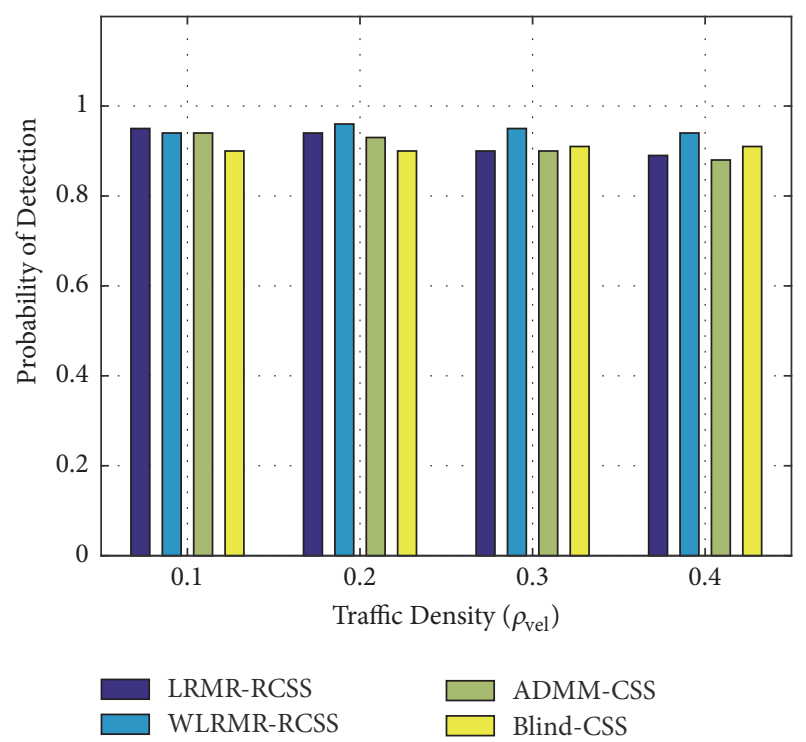

FIGURE 10: Comparison of the proposed LRMR-RCSS and WLRMRRCSS algorithms with Blind-CSS and ADMM-CSS algorithms at different traffic densities.

Figure 10 presents the performances of the four spectrum sensing algorithms as a function of the vehicle density at a road intersection in an urban environment. The traffic density is set to $0.1,0.2,0.3$, and $0.4 \mathrm{veh} / \mathrm{m} / \mathrm{l}$, denoting moderate, high, very high, and severe congestion traffic conditions, respectively. After a period of observation, it shows that the performance of our proposed WLRMR-RCSS algorithm remains stable under different traffic conditions. Our proposed WLRMR-RCSS algorithm outperforms the algorithms Blind-CSS and ADMM-CSS in the dense traffic environment. It is observed that our proposed LRMRRCSS algorithm is more suitable for sparse or even moderate traffic conditions. The performance of our proposed WLRMR-RCSS algorithm increases slightly from moderate traffic conditions to high traffic conditions. The improved SU diversity introduces this slight increase because of the increased number of cooperative vehicles. Of course, the diversity would not continually increase. This fact has been verified under the senior traffic condition, as the detection probability is not higher. When traffic is congested, our proposed algorithm is not affected. In such a scenario, the detection performance of ADMM-CSS algorithms would be reduced due to the increase of SUs. This indicates that our proposed WLRMR-RCSS algorithm is appropriate for dense vehicular environments.

According to Figure 11, we record the number of the selected cooperative SUs for WLRMR-RCSS at different traffic densities during our simulation process. We find that the number varies. This indicates that the proposed WLRMR-RCSS algorithm can adapt to various traffic densities as the number of cooperative users in the dense vehicular environment is not fixed but rather is altered according to the spatial distribution of vehicles. The number of selected cooperative SUs is not high in the high 


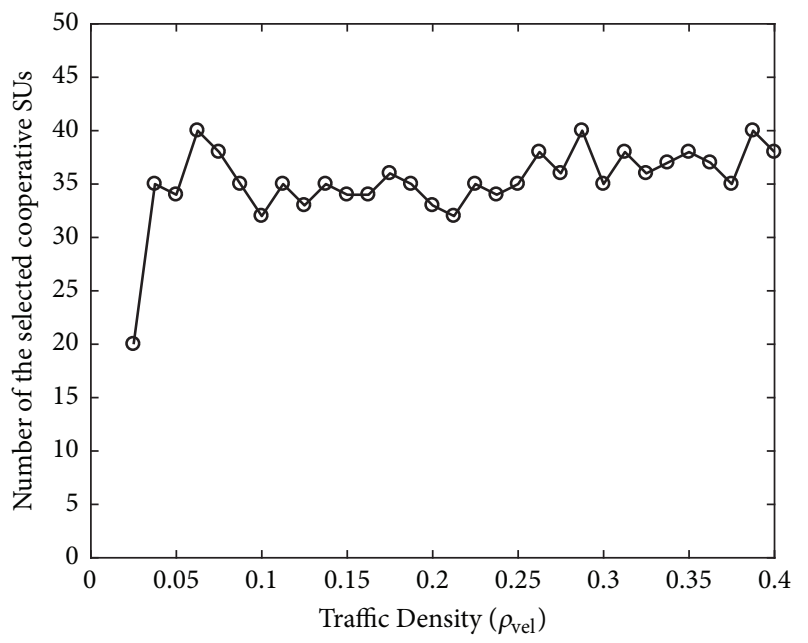

FIGURE 11: Number of the selected cooperative SUs for WLRMRRCSS at different traffic densities.

traffic density environment. That means that the selection of cooperative SUs in the WLRMR-RCSS algorithm could reduce the overhead in dense cognitive vehicular networks.

\section{Conclusions}

This work investigates the issue of robust and effective cooperative spectrum sensing in cognitive vehicular networks. We establish a robust spectrum sensing algorithm, LRMR-RCSS, to eliminate the negative impact of corrupted sensing data. In addition, we extend our robust cooperative spectrum sensing algorithm WLRMR-RCSS while utilizing cooperative diversity into dense CVNs. In the WLRMR-RCSS algorithm, we introduce a correlation-aware SU selection and weight assignment scheme to reduce the overhead of cooperative vehicles. The WLRMR-RCSS algorithm has realized cooperative SU diversity, and the historical information on vehicle reputation is not needed. Simulation results demonstrate that the proposed robust sensing WLRMRRCSS algorithm can achieve stable and competitive performance. The complexity of WLRMR-RCSS is not high. In practice, the cognitive vehicles are often caught in traffic jams due to road accidents or morning and evening peak traffic times. Our proposed WLRMR-RCSS algorithm could specifically be applied to dense traffic environments. These algorithms are performed by the vehicles which allows to discover white channels accurately. As a consequence, a greater number of vehicles are allowed to communicate safety and nonsafety information, which can prove diversified applications in areas that normally experience heavy traffic.

\section{Data Availability}

The data used to support the findings of this study are available from the corresponding author upon request.

\section{Disclosure}

This paper was presented in part at the IEEE/CIC International Conference on Communications in China, Qingdao, China, Oct. 22-24, 2017. This is an extended version.

\section{Conflicts of Interest}

The authors declare that there are no conflicts of interest regarding the publication of this paper.

\section{Acknowledgments}

This work was supported in part by the Chinese National Nature Science Foundation under Grants 61571062 and 61271177.

\section{References}

[1] World Health Statistics, World Health Organizaton, 2016.

[2] "IEEE standard for wireless access in vehicular environments (WAVE) - multi-channel operation," IEEE Std 1609.42016 (Revision of IEEE Std 1609.4-2010), pp. 1-206, 2016.

[3] C. Campolo and A. Molinaro, "Multichannel communications in vehicular Ad Hoc networks: a survey," IEEE Communications Magazine, vol. 51, no. 5, pp. 158-169, 2013.

[4] X. Yin, X. Ma, K. S. Trivedi, and A. Vinel, "Performance and reliability evaluation of BSM broadcasting in DSRC with multi-channel schemes," Institute of Electrical and Electronics Engineers. Transactions on Computers, vol. 63, no. 12, pp. 31013113, 2014.

[5] K. Xiong, X. Chen, L. Rao, X. Liu, and Y. Yao, "Solving the performance puzzle of DSRC multi-channel operations," in Proceedings of the IEEE International Conference on Communications, ICC 2015, pp. 3843-3848, June 2015.

[6] X. Ma, J. Zhang, X. Yin, and K. S. Trivedi, "Design and analysis of a robust broadcast scheme for VANET safety-related services," IEEE Transactions on Vehicular Technology, vol. 61, no. 1, pp. 46-61, 2012.

[7] Z. Wang and M. Hassan, "How much of dsrc is available for non-safety use?" in Proceedings of the 5th ACM International Workshop on VehiculAr Inter-NETworking (VANET '08), pp. 2329, ACM, New York, NY, USA, September 2008.

[8] S. Mumtaz, K. M. S. Huq, M. I. Ashraf, J. Rodriguez, V. Monteiro, and C. Politis, "Cognitive vehicular communication for 5G," IEEE Communications Magazine, vol. 53, no. 7, pp. 109116, 2015.

[9] K. D. Singh, P. Rawat, and J.-M. Bonnin, "Cognitive radio for vehicular ad hoc networks (CR-VANETs): approaches and challenges," EURASIP Journal on Wireless Communications and Networking, vol. 2014, article 49, 2014.

[10] M. di Felice, K. R. Chowdhury, and L. Bononi, "Cooperative spectrum management in cognitive vehicular Ad Hoc networks," in Proceedings of the IEEE Vehicular Networking Conference (VNC '11), pp. 47-54, November 2011.

[11] A. J. Ghandour, K. Fawaz, H. Artail, M. Di Felice, and L. Bononi, "Improving vehicular safety message delivery through the implementation of a cognitive vehicular network," Ad Hoc Networks, vol. 11, no. 8, pp. 2408-2422, 2013. 
[12] X. Y. Wang and P.-H. Ho, "A novel sensing coordination framework for CR-VANETs," IEEE Transactions on Vehicular Technology, vol. 59, no. 4, pp. 1936-1948, 2010.

[13] H. Kremo, O. Altintas, H. Tanaka, M. Kitamura, K. Inage, and T. Fujii, "Cooperative spectrum sensing in the vehicular environment: an experimental evaluation," in Proceedings of the 2014 IEEE Vehicular Technology Conference (VTC 2014-Spring), pp. 1-5, Seoul, South Korea, May 2014.

[14] M. Di Felice, A. J. Ghandhour, H. Artail, and L. Bononi, "Integrating spectrum database and cooperative sensing for cognitive vehicular networks," in Proceedings of the 2013 IEEE 78th Vehicular Technology Conference (VTC Fall), pp. 1-7, Las Vegas, NV, USA, September 2013.

[15] H. Li and D. K. Irick, "Collaborative spectrum sensing in cognitive radio vehicular ad hoc networks: belief propagation on highway," in Proceedings of the IEEE 71st Vehicular Technology Conference (VTC '10), pp. 1-5, Taipei, Taiwan, May 2010.

[16] B. Aygun and A. M. Wyglinski, "A voting-based distributed cooperative spectrum sensing strategy for connected vehicles," IEEE Transactions on Vehicular Technology, vol. 66, no. 6, pp. 5109-5121, 2017.

[17] C. Chembe, R. M. Noor, I. Ahmedy, M. Oche, D. Kunda, and C. H. Liu, "Spectrum sensing in cognitive vehicular network: Stateof-Art, challenges and open issues," Computer Communications, vol. 97, pp. 15-30, 2017.

[18] Z. Wei, F. R. Yu, and A. Boukerche, "Cooperative spectrum sensing with trust assistance for cognitive radio vehicular ad hoc networks," in Proceedings of the 5th ACM Symposium on Development and Analysis of Intelligent Vehicular Networks and Applications, DIVANet 2015, pp. 27-33, November 2015.

[19] Z. Wei, F. R. Yu, H. Tang, C. Liang, and Q. Yan, "Securing cognitive radio vehicular Ad hoc networks with trusted lightweight cloud computing," in Proceedings of the 2016 IEEE Conference on Communications and Network Security (CNS), pp. 450-456, Philadelphia, PA, USA, October 2016.

[20] G. Ding, J. Wang, Q. Wu et al., "Robust spectrum sensing with crowd sensors," IEEE Transactions on Communications, vol. 62, no. 9, pp. 3129-3143, 2014.

[21] X. Liu, Z. Zeng, and C. Guo, "Robust cooperative spectrum sensing in dense cognitive vehicular networks," in Proceedings of the 2017 IEEE/CIC International Conference on Communications in China (ICCC), pp. 1-6, October 2017.

[22] Y. Al-Mathehaji, S. Boussakta, M. Johnston, and H. Fakhrey, "Defeating SSDF attacks with trusted nodes assistance in cognitive radio networks," IEEE Sensors Letters, vol. 1, no. 4, pp. 1-4, 2017.

[23] P. T. Fishburn, J. Golkar, and K. M. Taaffe, "Simulation of transportation systems," in Proceedings of the 1995 Winter Simulation Conference, WSC'95, pp. 51-54, December 1995.

[24] M. Lpezbenłtez and F. Casadevall, "Spectrum usage models for the analysis, design and simulation of cognitive radio networks," Lecture Notes in Computer Science, vol. 62, no. 5, pp. 2091-2104, 2012.

[25] R. k Lindquist, "FCC spectrum policy task force reports on progress, initiatives," in Proceedings of the QST, vol. 76, p. 76, 2004.

[26] S. Pagadarai, A. M. Wyglinski, and R. Vuyyuru, "Characterization of vacant UHF TV channels for vehicular dynamic spectrum access," in Proceedings of the 2009 IEEE Vehicular Networking Conference, VNC 2009, October 2009.

[27] W. Lee and D. Cho, "Concurrent spectrum sensing and data transmission scheme in a CR system," in Proceedings of the 2012 IEEE Wireless Communications and Networking Conference (WCNC), pp. 1326-1330, Paris, France, April 2012.

[28] Y. Pei, Y. Liang, K. C. Teh, and K. H. Li, "Energy-efficient design of sequential channel sensing in cognitive radio networks: optimal sensing strategy, power allocation, and sensing order," IEEE Journal on Selected Areas in Communications, vol. 29, no. 8, pp. 1648-1659, 2011.

[29] S. Chaudhari, J. Lunden, V. Koivunen, and H. V. Poor, “Cooperative sensing with imperfect reporting channels: hard decisions or soft decisions?" IEEE Transactions on Signal Processing, vol. 60, no. 1, pp. 18-28, 2012.

[30] X. Liu, Z. Zeng, C. Guo, and S. Zhu, "Performance analysis of spatial-temporal spectrum sensing for cognitive vehicular network," in Proceedings of the the 3rd ACM Workshop, pp. 16, Paderborn, Germany, July 2016.

[31] Y. Zhao, P. Paul, C. Xin, and M. Song, "Performance analysis of spectrum sensing with mobile SUs in cognitive radio networks," in Proceedings of the 2014 1st IEEE International Conference on Communications, ICC 2014, pp. 2761-2766, aus, June 2014.

[32] L. Zhang, G. Ding, Q. Wu, and F. Song, "Defending against byzantine attack in cooperative spectrum sensing: defense reference and performance analysis," IEEE Access, vol. 4, pp. 4011-4024, 2016.

[33] A. Vosoughi, J. R. Cavallaro, and A. Marshall, "Trust-aware consensus-inspired distributed cooperative spectrum sensing for cognitive radio Ad Hoc networks," IEEE Transactions on Cognitive Communications and Networking, vol. 2, no. 1, pp. 2437, 2016.

[34] L. Zhang, G. Ding, Q. Wu, Y. Zou, Z. Han, and J. Wang, "Byzantine attack and defense in cognitive radio networks: a survey," IEEE Communications Surveys \& Tutorials, vol. 17, no. 3, pp. 1342-1363, 2015.

[35] Z. Tian and G. B. Giannakis, "Compressed sensing for wideband cognitive radios," in Proceedings of the IEEE International Conference on Acoustics, Speech and Signal Processing (ICASSP '07), pp. IV1357-IV1360, Honolulu, Hawaii, USA, April 2007.

[36] Y. Wang, Z. Tian, and C. Feng, "Collecting detection diversity and complexity gains in cooperative spectrum sensing," IEEE Transactions on Wireless Communications, vol. 11, no. 8, pp. 2876-2883, 2012.

[37] Z. Qin, Y. Gao, and M. D. Plumbley, "Malicious user detection based on low-rank matrix completion in wideband spectrum sensing," IEEE Transactions on Signal Processing, vol. 66, no. 1, pp. 5-17, 2018.

[38] S. Boyd, N. Parikh, E. Chu, B. Peleato, and J. Eckstein, "Distributed optimization and statistical learning via the alternating direction method of multipliers," Foundations and Trends in Machine Learning, vol. 3, no. 1, pp. 1-122, 2010.

[39] Z. Zhang, A. Ganesh, X. Liang, and Y. Ma, "TILT: transform invariant low-rank textures," International Journal of Computer Vision, vol. 99, no. 1, pp. 1-24, 2012.

[40] X. Ren and Z. Lin, "Linearized alternating direction method with adaptive penalty and warm starts for fast solving transform invariant low-rank textures," International Journal of Computer Vision, vol. 104, no. 1, pp. 1-14, 2013. 
[41] C. Tang, P. Wang, C. Zhang, and W. Li, "Salient object detection via weighted low rank matrix recovery," IEEE Signal Processing Letters, vol. 24, no. 4, pp. 1-5, 2017.

[42] K. Baraka, L. Safatly, H. Artail, A. Ghandour, and A. El-Hajj, "An infrastructure-aided cooperative spectrum sensing scheme for vehicular ad hoc networks," Ad Hoc Networks, vol. 25, pp. 197-212, 2015.

[43] VISSIM, http://vision-traffic.ptvgroup.com/en-us/products/ ptv-vissim/. 


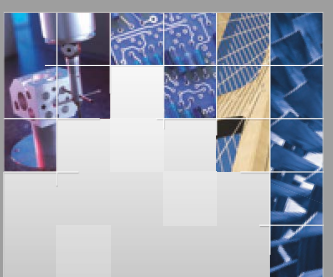

\section{Enfincering}
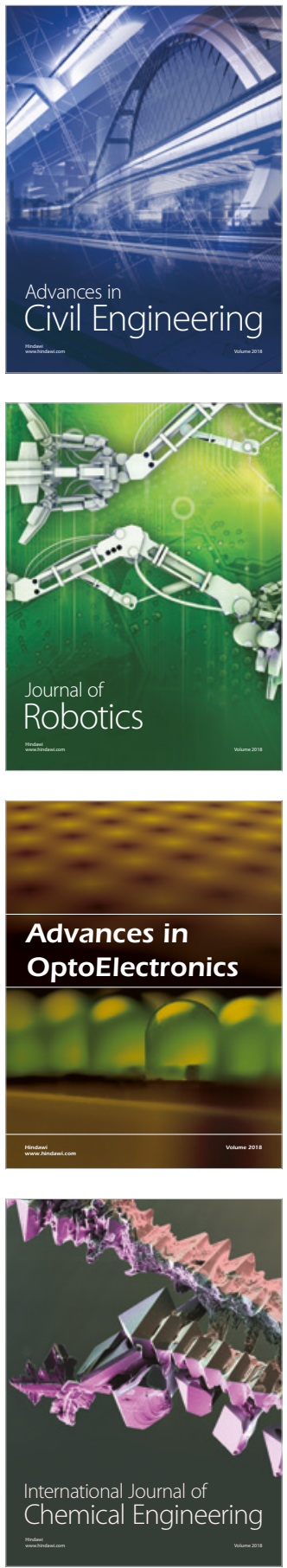

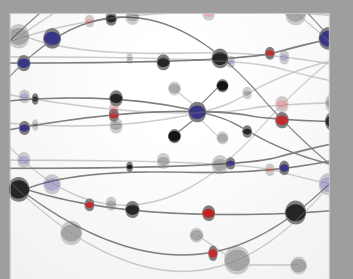

\section{Rotating \\ Machinery}

The Scientific World Journal

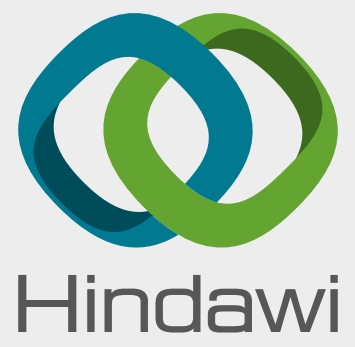

Submit your manuscripts at

www.hindawi.com
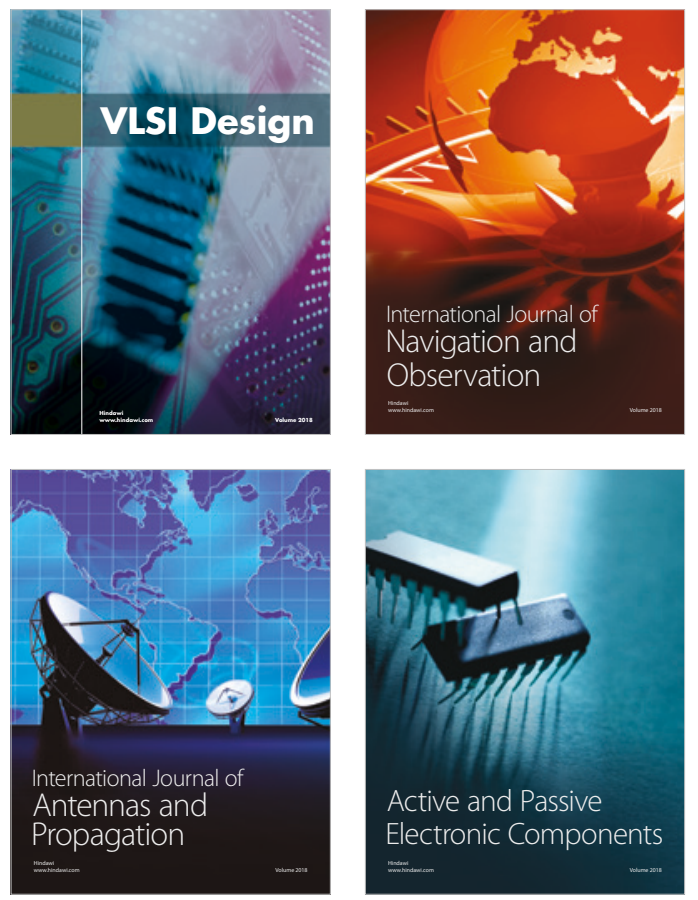
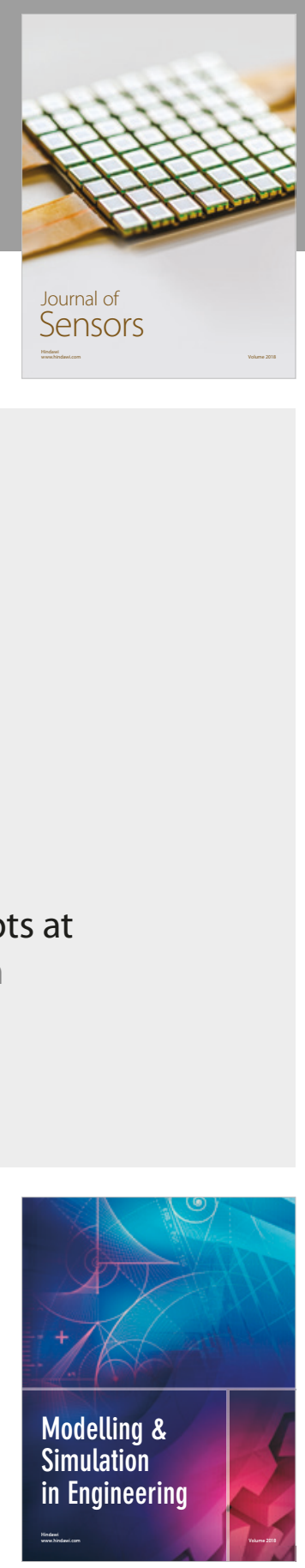

\section{Advances \\ Multimedia}
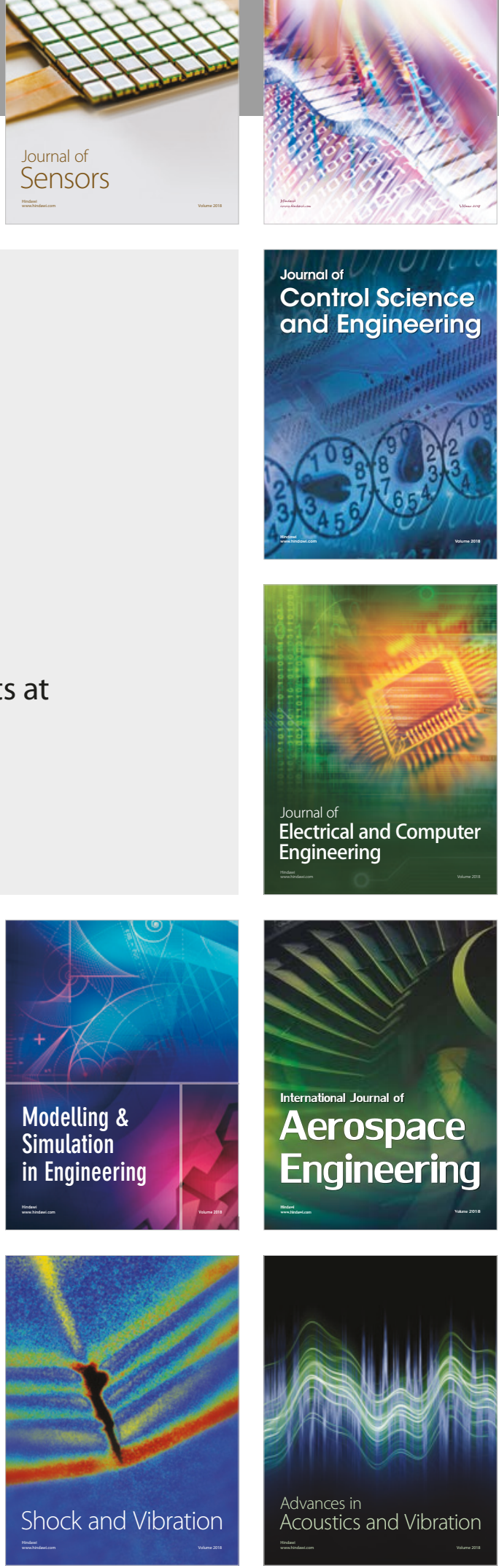